\title{
Etude comparée du début de la croissance chez divers génotypes de maïs
}

\author{
Robert BOURDU \& Nicole GREGORY \\ Laboratoire "Structure et Métabolisme des Plantes" associé au C.N.R.S. (LA 40), Université de Paris-Sud \\ (XI), F91405 Orsay Cédex
}

RÉSUMÉ On a suivi en conditions contrôlées le déroulement de la croissance (en poids de matière sèche) pendant les 12
premiers jours de germination de lots de semences de huit génotypes de mais.
Les observations mettent en évidence une crise de croissance qui se situe au $10^{\circ}$ jour au moment, semble-t-il,
où la plantule, ayant acquis un équipement photosynthétique suffisant, cesse d'utiliser majoritairement les
réserves de la semence. Des variations dans la vitesse et l'amplitude des phénomènes permettent des
comparaisons intervariétales. L'ensemble des résultats permet de dégager quelques concepts nouveaux
propres à être utilisés quantitativement pour les comparaisons au stade jeune.

Mots clés additionnels: Comparaisons intervariétales, stade jeune, germination, plantules, vigueur germinative, réserve.

Growth (dry weight) of germinating maize seedlings was studied during the first 12 days of culture under controlled conditions. Comparisons were made between eight varieties. Observations showed a growth crisis in all cases on the 10th day, at which time the seedlings have their own functional photosynthetic apparatus and have ceased to use their seed reserve. Variations in the rate and amplitude of the phenomenon provided a basis for intervarietal comparison. These results have contributed to the definition of some new concepts which can be useful for quantitative comparisons among varieties in the early stage of growth.

Additional key words: Intervarietal comparisons, early stage, germination plantlets, seedling vigour, storage.

\section{INTRODUCTION}

Toute plante, dans la période initiale de son développement, se trouve confrontée à 3 problèmes:

1) Elle doit puiser dans un réservoir qui lui est extéricur les ressources nécessaires à sa croissance initiale. Le réservoir peut ĉtre l'albumen pour l'embryon, les tissus d'un explantat pour une bouture ou le milieu synthétique de culture pour un développement in vitro. Ces ressources initiales peuvent occuper des organes anatomiquement intégrés à la jeune plante comme les cotylédons, mais les réserves, là aussi, y ont été déposées par l'activité d'une plante mère.

2) La jeune plantule doit créer assez vite les structures spécialisées aptes à lui faire acquérir une autonomie métabolique.

3) Le $3^{c}$ problème concerne précisément la liaison entre ics 2 précédents. Les ressources extérieures s'épuisent si elles ne sont pas entretenues; or le renouvellement n'est réalisé que dans les cas de cultures in vitro. La mise en place des structures d'autonomic (racines, feuilles et les systèmes de transport et de synthèse) suit une programmation qui est sous le double contrôle des propriétés génotypiques et des conditions ambiantes. Il est donc clair qu'entre la phase d'utilisation et celle de néosynthèse, se situe une période de transition cruciale pour la jeune plante. Comment se fait cette transition? Est-elle marquée par quelque manifestation particulière du développement? Enfin comment cette transition est-elle assurée par différents génotypes voisins? Nous présentons des résultats qui constituent des éléments de réponse à ces questions dans le cas du maïs.

Cette période transitoire est courte. Pour suivre son déroulement, il est nécessaire de déceler des variations du métabolisme suivant un rythme d'échantillonnage rapide. Les informations, cependant très précieuses, obtenues par DURE $(1960 a)$ ne permettent pas, avec des prélèvements tous les 2 jours, de mettre en évidence un phénomène fugace. Les courbes de croissance présentées par INGLE et al. (1964) ne dépassent pas 5 jours, ce qui est trop court ; seule la phase initiale de stricte utilisation des réserves de l'albumen est couverte par ce laps de temps. Les analyses de croissance effectuées par HUNT \& EVANS (1980) à partir de 
données anciennes permettent de comparer les plantes mais sur la période totale de la croissance (100 jours), ce qui limite la finesse des observations autour d'une phase précoce. Les meilleures données permettant d'ébaucher une réponse aux questions posées plus haut concernant le maïs ont été fournies par les travaux de COOPER \& MACDONALD (1970). Par des courbes de prise de poids de matière sèche, ces auteurs montrent qu'au $10^{\mathrm{c}}$ jour qui suit la germination, un ralentissement de la croissance est décelable.

L'étude de la croissance exprimée par la variation du poids de matière sèche que nous présentons met en évidence l'importance qualitative de l'accident de transition qui s'observe une dizaine de jours après la germination. L'étude rassemble un faisceau de données quantitatives qui doit permettre de formuler, en termes rigoureux, les notions de vigueur germinative (WHALLEY et al., 1966) et de vitesse de développement. Elle permet également d'évaluer le rôle, sur la production. finale, de la dépression, variable suivant les génotypes, décelée au cours des phases initiales de la croissance.

Les résultats font peu appel aux formules habituelles de «l'analyse de croissance » prises dans le sens traditionnel (RADFORD, 1967). Nous avons préféré introduire des observations et des concepts nouveaux qui se rapprochent plus, semble-t-il, des étapes métaboliques et morphologiques de la germination et du début de développement.

\section{MATÉRIEL ET MÉTHODES}

Les semences de maïs correspondant aux 8 génotypes suivants : $\mathrm{W}_{\mathrm{H}} \times \mathrm{W}_{\mathrm{J}} ; \mathrm{F}_{7} \times \mathrm{F}_{2} ; \mathrm{LG} 11 ; \mathrm{BIP}\left(\mathrm{W}_{529 \mathrm{~A}} \times \mathrm{F}_{2}\right)$; HTV 216 (= Bruex INRA 210); HS 222 ; INRA 250 et INRA 508 nous ont été fournies par la Station d'Amélioration des Plantes (INRA) de Mons-en-Chaussée.

Les semences, individuellement pesées, sont placées dans des godets remplis de vermiculite (gros module) et l'imbibition commence immédiatement $\left(\mathrm{t}_{0}\right)$ par apport d'eau. L'irrigation est maintenue à saturation par un système de réservoirs à trop plein dans lesquels sont placés les godets. Après $3 \mathrm{j}$ d'imbibition (jour 3 ) et jusqu'à la fin de la culture (12 j), l'cau est remplacée par une solution nutritive (solution $\mathrm{BB}^{\prime}$ de Coïc spéciale maïs diluće 2 fois). Par renouvellement de la solution tous les 3 jours, on obtient un pH constant et on évite un épuisement éventuel des constituants du milicu nutritif.

Dès la mise en culture $\left(t_{0}\right)$ jusqu'à la fin de l'expérience $\left(t_{12}\right)$ les plantes sont maintenues dans une enceinte à microclimat constant : $16 \mathrm{~h}$ de lumière par $24 \mathrm{~h}$. La lumière est fournie par 10 tubes TLS W34 (Philips) sans réflecteur. L'éclairement mesuré par thermopile à hauteur de l'apex des plantes est de $60 \mathrm{~W} \cdot \mathrm{m}^{-2}\left(=220 \mu \mathrm{E} . \mathrm{m}^{-2}, \mathrm{~s}^{-1}\right)$. Par un déplacement vertical des cultures, l'éclairement est maintenu constant au niveau des apex. La température est de $25^{\circ} \mathrm{C}$ pendant la période lumineuse et de $22^{\circ} \mathrm{C}$ pendant l'extinction des tubes. L'humidité relative est comprise entre 70 et 85 p. 100 .

Dans ces conditions, le développement des plantes se fait suivant un rythme décrit dans le tableau 1. Il permet un repérage de l'équivalence des stades et des âges dans nos conditions expérimentales. Suivant les habitudes des laboratoires, la littérature donne des informations soit en âge (jours, heures...) soit en stade de développement (nombre de feuilles...). Dans la suite des résultats, c'est toujours l'âge exprimé en jours qui sera utilisé pour décrire le déroulement du temps.
Dix plantes sont prélevées au hasard chaque jour (à $10 \mathrm{~h}$, c'est-à-dire après $4 \mathrm{~h}$ d'éclairement) et, dans certains cas, 2 fois par jour ( $10 \mathrm{~h}$ et $21 \mathrm{~h}$ ). L'heure exacte des prélèvements a été respectée car les variations de poids au cours de la journée peuvent être, pour ces jeunes plantes, très importantes (HUNT, 1980). Les plantes sont immédiatement disséquées en 2 parties: d'une part, la plantule (=les parties aériennes plus le système racinaire) et, d'autre part, les tissus de réserve et les téguments. Pour chaque germination, les pesées sont effectuées séparément. On peut ainsi rapporter le poids des prélèvements au poids initial connu de la semence. Les 2 parties sont desséchées à $80^{\circ} \mathrm{C}$ pendant $48 \mathrm{~h}$ puis pesées. Les expériences ont été répétées 2 ou 3 fois.

\section{RÉSULTATS}

Le paramètre de croissance utilisé est toujours le poids de matière sèche. La figure 1 montre l'évolution de cette valeur en fonction du temps pour la jeune plante (P) d'un des génotypes étudiés (INRA 250) au cours des 12 premiers jours de croissance. La somme de température, compte tenu de la régulation thermique de la salle de culture, est facile à calculer par la formule $0,5\left(t_{x}+t_{N}\right)-10$. Elle représente $13,5^{\circ} \mathrm{C} / \mathrm{j}$. L'arrêt de l'expérience a donc eu lieu pour $\Sigma_{t}=162^{\circ}$. L'état morphologique de la plante est indiqué dans le tableau 1.

La figure $1 a$ montre également la cinétique de décroissance en poids de matière sèche de tout ce qui, dans la germination, n'est pas la plantule : téguments, albumen, scutellum. On constate que la décroissance n'est pas régulière. Dans une première phase, jusqu'au $6^{\mathrm{e}} \mathrm{j}$., elle va s'accélérant. A partir du $6^{\mathrm{e}} \mathrm{j}$., elle se ralentit et tend vers une limite. La valeur ( $\mathrm{r}$ ) de ce résidu correspond à ce qui, des réserves et téguments, ne sera pas utilisé pour la croissance de la plantule. Deux remarques peuvent être faites au sujet de ce résidu :

- son poids représente dans le cas de ce génotype 13,5 p. $100 \mathrm{du}$ poids initial. Ce résidu a une importance pondérale relative très variable suivant les cas, les valeurs allant d'environ 12 p. 100 (HS 222) à environ 28 p. 100 (LG 11). Il existe une corrélation entre la valeur relative des résidus et les poids initiaux des semences. Les semences de HS 222 étaient les plus légères $(210 \mathrm{mg})$ et celles de LG 11 les plus lourdes $(320 \mathrm{mg})$. Les autres génotypes fournissent des semences de poids intermédiaire.

- Ce résidu n'est pas seulement formé des seuls téguments inutilisés car le poids de ceux-ci ne représente que 5 à 8 p. 100 du total de la semence. On peut donc considérer que cette matière résiduelle renferme des tissus de réserve non totalement épuisés. Dans la suite des comparaisons intergénotypiques, la valeur pondérale des réserves sera calculée en soustrayant du poids total (albumen + scutellum + téguments) cette partie inutilisée. Ainsi dans les courbes de décroissance des réserves, on atteindra les valeurs nulles, non parce que l'ensemble a été employé ou épuisé, mais parce que la notion de réserve a été restreinte à la masse de métabolites utilisables et, à terme, utilisés, bien entendu dans nos conditions expérimentales.

L'analyse de la croissance (et de la décroissance des «réserves utiles») peut être poussée plus loin en passant en coordonnées semi-logarithmiques, ce qui est fait dans la figure $1 b$ pour le génotype INRA 250 , toujours pris en exemple. On constate que la courbe de croissance de la jeune plante $(P)$ se divise en 2 parties: jusqu'au $6^{\mathrm{e}}$ jour environ, le taux de croissance (= vitesse relative de crois- 


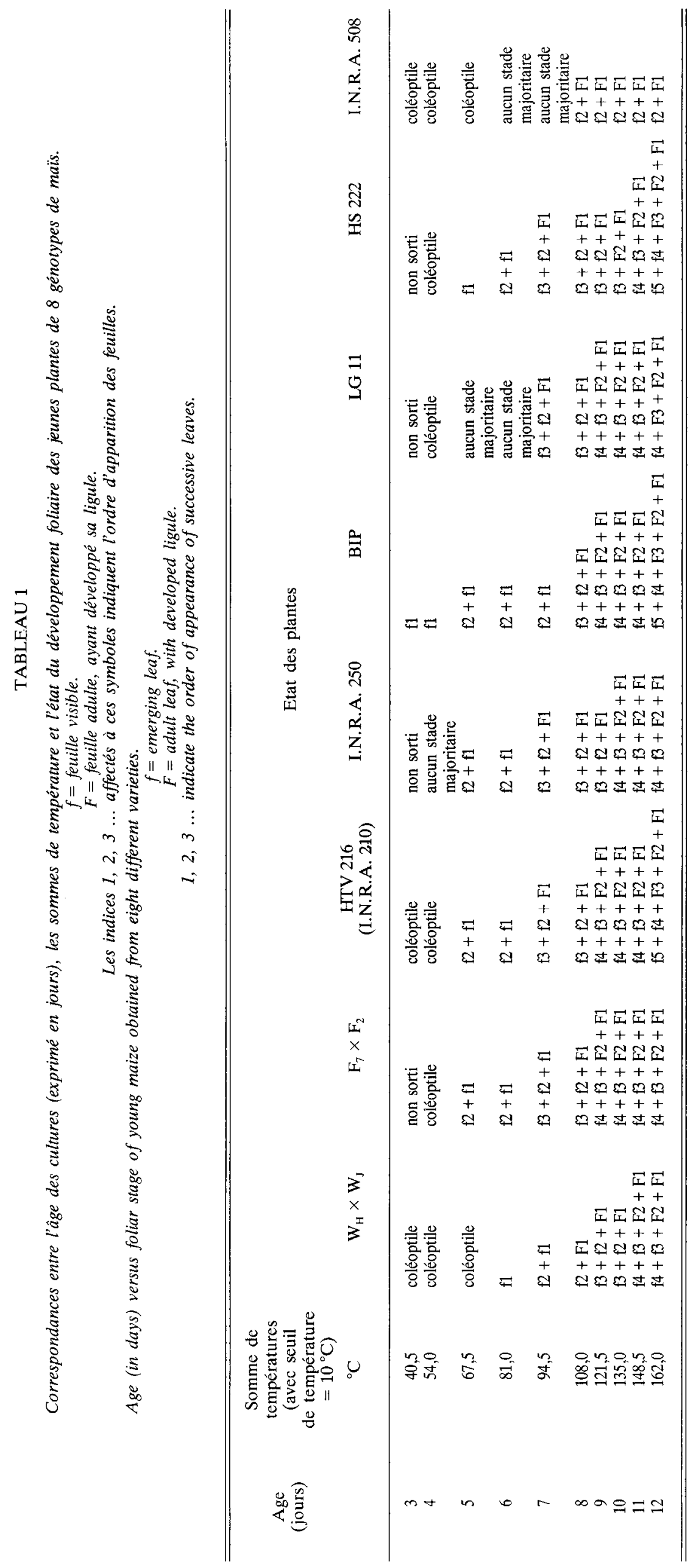




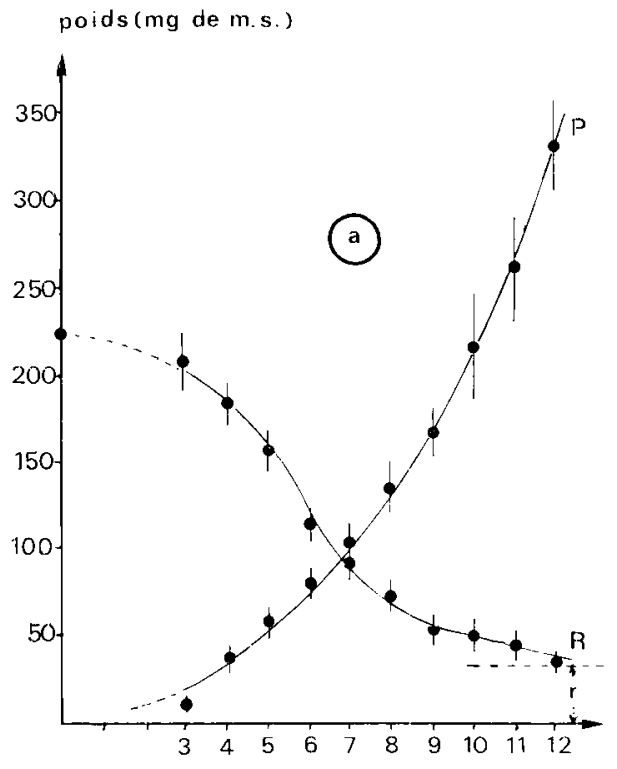

$\log$ poids de m.s.

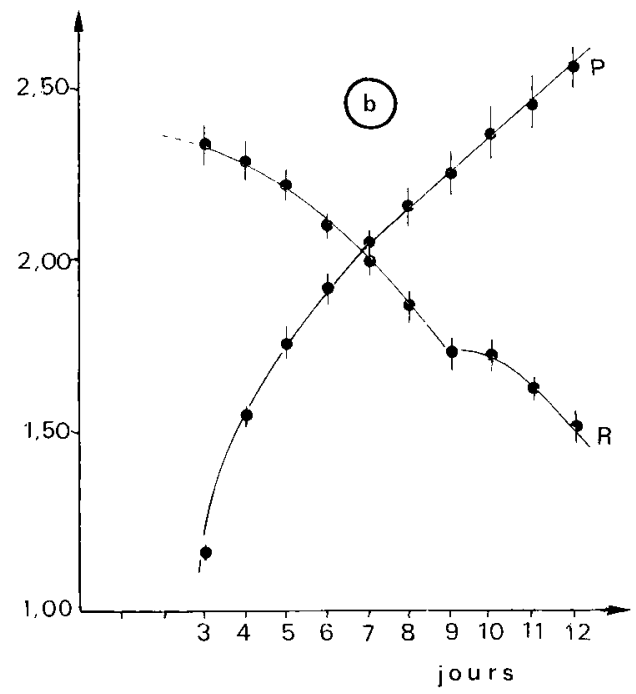

Figure 1

Courbes de croissance et de disparition des réserves pour de jeunes plantes de maïs (I.N.R.A. 250).

$P$ : plantule.

$R:$ organes de réserve (albumen + scutellum) + téguments.

$r:$ partie des réserves inexploitées.

a: ordonnée décimale.

$b$ : ordonnée logarithmique.

Growth curves and reserve decreases for seedlings of maize (I.N.R.A. 250).

$P$ : Seedling.

$R:$ Endosperm + scutellum + teguments

$r:$ untapped reserves.

a: decimal scale.

$b$ : semi-logarithmic scale.

sance $\neq$ vitesse de croissance) décroît régulièrement. Le taux de croissance est $\rho=\mathrm{dp} / \mathrm{P}_{\mathrm{o}}{ }^{\mathrm{dt}}$ où dp est la prise de poids de matière sèche à partir d'un poids initial $\mathrm{P}_{0}$ et pendant un espace de temps dt. A partir du $6^{\mathrm{e}}$ jour, ce taux se stabilise et on atteint une phase d'allure exponentielle qui, sur ce type de graphique, se traduit par une droite dont la pente égale $\rho$.

La courbe de décroissance des "réserves utiles" se fait suivant un taux qui s'accélère jusqu'au $8^{\mathrm{c}}$ ou $9^{\mathrm{c}}$ jour. On observe entre le $9^{\mathrm{e}}$ et $10^{\mathrm{e}} \mathrm{j}$ un arrêt de cette disparition des réserves qui reprend par la suite. Cette cassure dans la courbe décroissante était déjà visible mais moins nettement exploitable dans le système de coordonnées décimales de la figure $1 a$.

C'est parce que les comparaisons de taux (croissance ou décroissance) sont très largement facilitées par la représentation semi-logarithmique que c'est elle que nous avons adoptéc pour les confrontations intergénotypiques: figures 2 et 3 pour les croissances de plantules, figures 4 et 5 pour les disparitions des réserves « utiles » correspondantes.

Dans les figures 2 et 3 , on a regroupé les génotypes en 2 catégories : les 4 de la figure 2 présentent un palier assez net au $10^{\mathrm{e}} \mathrm{j}$ de leur croissance, les 4 de la figure 3 ne se signalent pas (ou très peu pour BIP) par cet accident. Dans la figure 2 on note que les génotypes ont été disposés de gauche à droite et de haut en bas dans un ordre d'importance décroissante de cet accident. Ainsi l'accident le mieux marqué, au point qu'une ébauche de dépression peut être déccléc, est enregistré pour $\mathrm{F}_{7} \times \mathrm{F}_{2} ;$ l'accident significatif le moins profond étant relevé chez HS 222 . Il est remarquable que, pour ces 4 variétés (plus BIP de la fig. 3), l'arrêt de la croissance ou unc diminution brutale et temporaire du taux de croissance a lieu, toujours dans nos conditions expérimentales, entre le $9^{c}$ et le $10^{c}$ jour. Dans tous les cas, l'événement correspond à une utilisation des réserves « utiles » comprise entre 80 et 90 p. 100 de sa valeur initiale.

Si l'on considère les courbes de la figure 3 , où l'on retrouve INRA 250 déjà illustré à la figure $1 b$ mais replacé ici par souci de comparaison, on constate que, pour 3 génotypes au moins $\left(\mathrm{W}_{\mathrm{H}} \times \mathrm{W}_{\mathrm{J}}\right.$, HTV 216 et INRA 250), après unc période de $6 \mathrm{j}$ environ qui correspond à une diminution régulière du taux de croissance, on atteint un état stationnaire qui se traduit par des droites. On peut noter l'cxacte similitude des graphes illustrant la croissance des génotypes HTV 216 et INRA 250.

En comparant les pentes des droites (dans la mesure où les points expérimentaux le permettent) dans la période qui succède à l'accident de croissance du $10^{\mathrm{e}} \mathrm{j}$, on constate que les pentes les plus fortes (les taux de croissance les plus élevés) sont justement celles enregistrées avec les génotypes qui ont manifesté l'accident le plus profond: $\mathrm{F}_{7} \times \mathrm{F}_{2}$ et INRA 508. Les taux de croissance stationnaires s'établissent ensuite suivant l'ordre décroissant : BIP, puis 4 génotypes à taux de croissance égaux: HS 222, LG 11, HTV 216 et INRA 250 , enfin $W_{H} \times W_{J}$ qui présente un taux de croissance nettement plus faible. Mais si la valeur du taux constant permet ce classement et débouche sur une interprétation possible quant à la vitesse de mise en place de la jeunc plante, il faut aussi tenir compte, dans ce type de comparaison, de 3 éléments : le taux initial (pente à l'origine) et il semble très élevé pour $\mathrm{W}_{\mathrm{H}} \times \mathrm{W}_{\mathrm{J}}$, par exemple ; la concavité de la courbe dans sa phase initiale (on peut comparer $\mathrm{F}_{7} \times \mathrm{F}_{2}, \mathrm{~W}_{\mathrm{H}} \times \mathrm{W}_{\mathrm{J}}$, d'une part, avec LG 11, HS 222 et BIP, d'autre part) ; le retard, enfin, causé par l'accident du $10^{\mathrm{e}} \mathrm{j}$ dont on a déjà signalé le caractère plus ou moins marqué suivant les génotypes.

$\mathrm{Si}$ l'on considère maintenant les pertes en réserves illustrées pour les mêmes génotypes par les figures 4 et 5 , on constate qu'on retrouve à la même date, le $10^{\mathrm{e}} \mathrm{j}$, un phénomène symétrique à l'arrêt ou au ralentissement de la croissance. C'est dans les 2 génotypes qui présentent un accident de croissance important $\left(\mathrm{F}_{7} \times \mathrm{F}_{2}\right.$ et INRA 508) que l'on enregistre l'arrêt le plus net dans la décroissance des réserves. D'autre part, l'extrême similitude des courbes de croissance des génotypes HTV 216 et INRA 250 se retrouve dans la disparition des réserves.

On peut pousser plus loin les comparaisons entre les expériences et obtenir un complément d'information en étudiant l'évolution du rapport entre le poids total (matière 
sèche) à un moment donné et le poids initial (exprimé également en matière sèche). L'évolution de ce rapport est illustré pour les 8 génotypes dans la figure 6 . Les courbes, comme on pouvait s'y attendre, présentent 2 parties : une phase initiale au cours de laquelle les pertes ne sont pas compensées par les gains photosynthétiques et le rapport est inférieur à l'unité et une $2^{\mathrm{e}}$ phase où les gains compensent et dépassent les pertes éventuelles; le bilan devient positif et les valeurs sont supérieures à 1 . On note que l'accident de croissance décrit par les représentations précédentes se retrouve toujours (sauf pour HS 222) dans les représentations globales actuelles et qu'il se situe toujours immédiatement après le passage de la barre du bilan nul $($ rapport $=1)$ ).

Que cet accident se révèle par ce type de représentation où, seules, les valeurs globales des germinations sont prises en compte, signifie que le bilan entre les arrêts (ou ralentissements) de croissance et les paliers concomitants de l'utilisation des réserves reste négatif. Cet aspect sera soumis à discussion ultérieurement mais, dès à présent, on peut observer que le retard pris par l'importance de ce bilan négatif cst variable selon les cas. Les pointillés, sur la figure 6, tentent d'extrapoler ce que pourrait être la courbe, en effaçant l'accident de croissance, et permettent peut être de quantifier ce retard en évaluant la différence entre les valeurs réelles enregistrécs au $11^{\mathrm{e}}$ ou $12^{\mathrm{e}} \mathrm{j}$ et les valeurs extrapolées.
D'autres paramètres des courbes de la figure 6 méritent d'être signalés dans la mesure où ils traduisent des différences de comportement. On peut relever, en particulier, la forme de la courbe dans sa partie inférieure à 1 et la date à laquelle se réalise le passage des valeurs inférieures à 1 aux valcurs supérieures à l'unité. La concavité, dans sa phase initiale, exprime l'importance du déficit durant la période de germination où les gains restent inférieurs au coût énergétique des transformations et transferts métaboliques. Les valeurs les plus éloignées de l'unité sont obtenues avec les semences $F_{7} \times F_{2}$, les plus proches de 1 , avec celles de INRA 508.

La date de passage des valeurs inférieures à 1 , aux valeurs supérieures à 1 , exprime la précocité du phénomène. La précision n'est pas très grande, cependant on peut classer les cas en 2 groupes: ceux pour lesquels la courbe passe la droite d'ordonnée 1 entre le $8^{\mathrm{e}}$ et $9^{\mathrm{e}} \mathrm{j}\left(\mathrm{F}_{7} \times \mathrm{F}_{2}\right.$, BIP, LG 11, HTV 216, HS 222) et ceux pour lesquels cet événement a lieu entre le $9^{\mathrm{e}}$ et $10^{\mathrm{c}} \mathrm{j}$ (INRA 508, INRA 250, $\mathrm{W}_{\mathrm{H}} \times \mathrm{W}_{\mathrm{J}}$ ). La similitude des courbes, jusqu'ici signalée pour HTV 216 et INRA 250 , marque pour ce paramètre de précocité une différence qui va dans le même sens que la différence d'indice de précocité (240 et 300 ) qu'on attribue à ces 2 génotypes. Une donnće caractéristique des $1^{\text {ers }}$ stades de la vie de la jeune plante peut être l'importance cumulée du déficit initial, valeur qui intègre les 2 informations précédentes: la concavité de la courbe et la durée de la phase. On

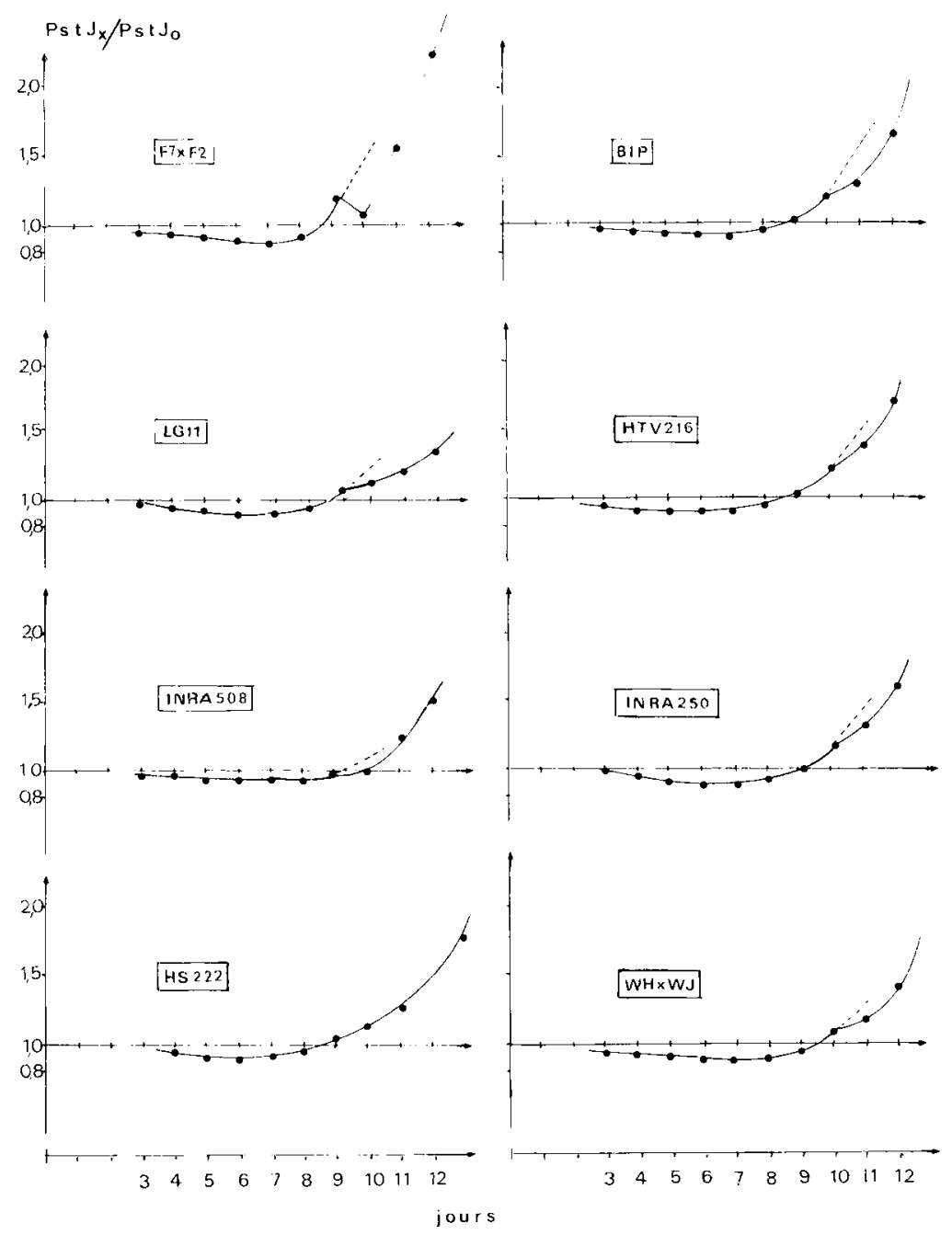

Figure 6

Evolution du rapport: $P d s$ matière seche totale $\left(P_{s} t J_{x}\right) / P d s$ matière sèche initiale de la semence $\left(P_{s} t J_{o}\right)$. Comparaison entre 8 génotypes de maïs.

Variation of the ratio: total dry weight, day $J x\left(P_{s} t J_{x}\right) /$ total initial dry weight $\left(P_{s} t J_{o}\right)$. Comparison between 8 maize varieties. 
peut rendre compte de cette valeur intégrée en mesurant la surface $(S)$ de l'aire comprise entre la courbe (partie $<1$ ) et l'axe d'ordonnée 1.

Le tableau 2 donne en valeurs arbitraires cette surface $S$ pour les différents génotypes. On peut répartir les valeurs obtenues en 3 groupes :

- les valeurs élevées $(\mathrm{S}>70)$ pour $\mathrm{W}_{\mathrm{H}} \times \mathrm{W}_{\mathrm{J}}, \mathrm{F}_{7} \times \mathrm{F}_{2}$ puis INRA 250 et HTV 216,

- les valeurs moyennes $(S \simeq 60$ ) pour $L G 11$, INRA 508 et BIP.

- une valeur faible ( $S=48)$, très inférieure à celles des deux groupes précédents, pour HS 222.

On peut estimer la dépense que représente pour une jeune plantule sa croissance dans ses phases initiales, alors que l'apport énergétique photosynthétique ne se manifeste pas ou ne s'est pas efficacement établi. Pour cela, il est possible, à partir des données exposées plus haut, de calculer un rapport qu'on obtient en divisant la prise de poids (en matière sèche) de la plantule $(\mathrm{dP})$ par la perte de poids (en matière sèche) de l'ensemble des réserves (dR) pendant le même laps de temps. A l'origine, avant toute activité photosynthétique, ce rapport $\mathrm{dP} / \mathrm{dR}$ mesure le coût du transfert des métabolites des réservoirs vers les méristèmes. Mais très tôt, cette valeur s'élève et son évolution au cours du temps traduit la vitesse de mise en place d'un système autotrophique. Le tableau 3 indique les valeurs $\mathrm{dP} / \mathrm{dR}$ pour les 8 variétés étudiées et durant les $12 \mathrm{j}$ de l'expérience.

On constate qu'au début de la croissance les valeurs sont assez dissemblables: élevées $(>0,50)$ pour INRA 508, LG 11, INRA 250 et $F_{7} \times F_{2}$, moyennes pour HTV 216 et $W_{H} \times W_{J}$, très faible, enfin, pour BIP. (L'expérience avec HS 222 n'ayant commencée que le $4^{e} \mathrm{j}$, il n'est pas possible de classer ce génotype parmi les autres, cependant la valeur élevée du $4^{\mathrm{e}} \mathrm{j}(0,73)$ permet de penser qu'il devrait figurer dans le groupe à rapport $\mathrm{dP} / \mathrm{dR}>0,5)$.

La lecture du tableau 3 nous informe donc sur la variabilité du coût initial de la croissance. Il nous indique aussi qu'au cours du temps on peut distinguer 3 phases:

- initialement et jusqu'au $6^{\mathrm{e}}$ ou $7^{\mathrm{e}} \mathrm{j}$, la variabilité intergénotypique s'estompe graduellement ;

- aux $7^{\mathrm{e}}, 8^{\mathrm{e}}$ et $9^{\mathrm{e}}$ jours, le rapport atteint, en s'approchant de l'unité, une uniformité assez remarquable entre les génotypes ;

- enfin, par la suite, des différences non négligeables réapparaissent.
TABLEAU 2

Surfaces ( $S$, en valeurs arbitraires) de l'aire comprise entre la courbe (fig. 6) dans sa partie < 1 et l'axe d'ordonnée 1 pour des semences provenant de 8 génotypes de maïs.

Area ( $S$, in arbitrary values) of the area between the curve (fig. 6) in its part $<1$ and the $Y$-axis $(=1)$.

\begin{tabular}{ll}
\hline \hline Génotypes & $\mathrm{S}$ \\
\hline $\mathrm{W}_{\mathrm{H}} \times \mathrm{W}_{\mathrm{J}}$ & 82 \\
$\mathrm{~F}_{7} \times \mathrm{F}_{2}$ & 77 \\
I.N.R.A. 250 & 75 \\
HTV 216 & 72 \\
LG 11 & 63 \\
I.N.R.A. 508 & 60 \\
BIP & 58 \\
HS 222 & 48 \\
\hline
\end{tabular}

La vitesse de mise en place d'un équipement photosynthétique peut être exprimée non seulement en fonction du temps mais aussi en fonction de la quantité de réserves nécessaires pour atteindre la pleine efficacité de photosynthèse. C'est ce que montre la figure 7. Dans ces graphiques, la croissance de la plantule n'est pas exprimée en fonction du temps mais en fonction du pourcentage des réserves «utiles» consommées. On remarque que, dans tous les cas, les points expérimentaux sont d'abord reliés par une droite passant par l'origine des axes et qu'ensuite la relation linéaire disparaît. Deux paramètres sont intéressants à relever :

- le pourcentage de réserves utilisées lorsque la croissance de la plantule cesse d'être linéairement reliée à cette utilisation (valeur dans le cercle et flèche sur la figure 7), - la pente $(\mathrm{p})$ de cette droite initiale.

On constate qu'il faut que de 70 à 75 p. 100 des réserves soient utilisées chez $F_{7} \times F_{2}$, INRA 508, HS 222, INRA 250 pour que la croissance de la plantule échappe au contrôle dû, semble-t-il, à la perte des ressources des tissus d'accumulation. Par contre, dès que 40,50 ou 55 p. 100 des réserves sont exploitées, chez HTV 216, LG 11 et BIP, la croissance de la plantule n'est plus linéairement corrélée à la perte en réserves.

On ne peut pas parler ici de «précocité » au sens où ce concept introduit la notion de temps. On préfère employer le concept d'«efficacité d'utilisation» dans le sens où une

\section{TABLEAU 3}

Evolution du rapport gain/perte pour 8 génotypes de maïs durant les 12 premiers jours de la croissance. Variation of the gain/loss ratio for eight varieties of maize during the first 12 days of growth.

\begin{tabular}{|c|c|c|c|c|c|c|c|c|}
\hline $\begin{array}{c}\text { Nombre de jours } \\
\text { après } \\
\text { l'imbibition }\end{array}$ & $\mathbf{F}_{7} \times \mathrm{F}_{2}$ & LG 11 & I.N.R.A. 508 & HS 222 & $\mathrm{BIP}$ & HTV 216 & I.N.R.A. 250 & $\mathrm{~W}_{\mathrm{H}} \times \mathrm{W}_{\mathrm{J}}$ \\
\hline 3 & 0,50 & 0,58 & 0,61 & - & 0,14 & 0,46 & 0,52 & 0,35 \\
\hline 4 & 0,62 & 0,36 & 1,00 & 0,73 & 0,14 & 0,51 & 0,76 & 0,46 \\
\hline 5 & 0,65 & 0,68 & 0,85 & 0,66 & 0,64 & 0,67 & 0,77 & 0,67 \\
\hline 6 & 0,69 & 0,67 & 0,80 & 0,76 & 0,68 & 0,73 & 0,72 & 0,73 \\
\hline 8 & 0,82 & 0,84 & 0,89 & 0,85 & 0,84 & 0,88 & 0,87 & 0,82 \\
\hline 9 & 1,29 & 1,07 & 1,00 & 1,01 & 0,96 & 1,02 & 0,95 & 0,94 \\
\hline 10 & - & 0,99 & 1,48 & 1,11 & 1,16 & 1,32 & 1,21 & 1,13 \\
\hline 11 & 1,69 & 1,38 & 2,02 & 1,30 & 1,38 & 1,48 & 1,39 & $\begin{array}{l}1,12 \\
1,35\end{array}$ \\
\hline 12 & 2,50 & 1,51 & 2,04 & 1,96 & 1,88 & 1,88 & 1,70 & 1,63 \\
\hline
\end{tabular}



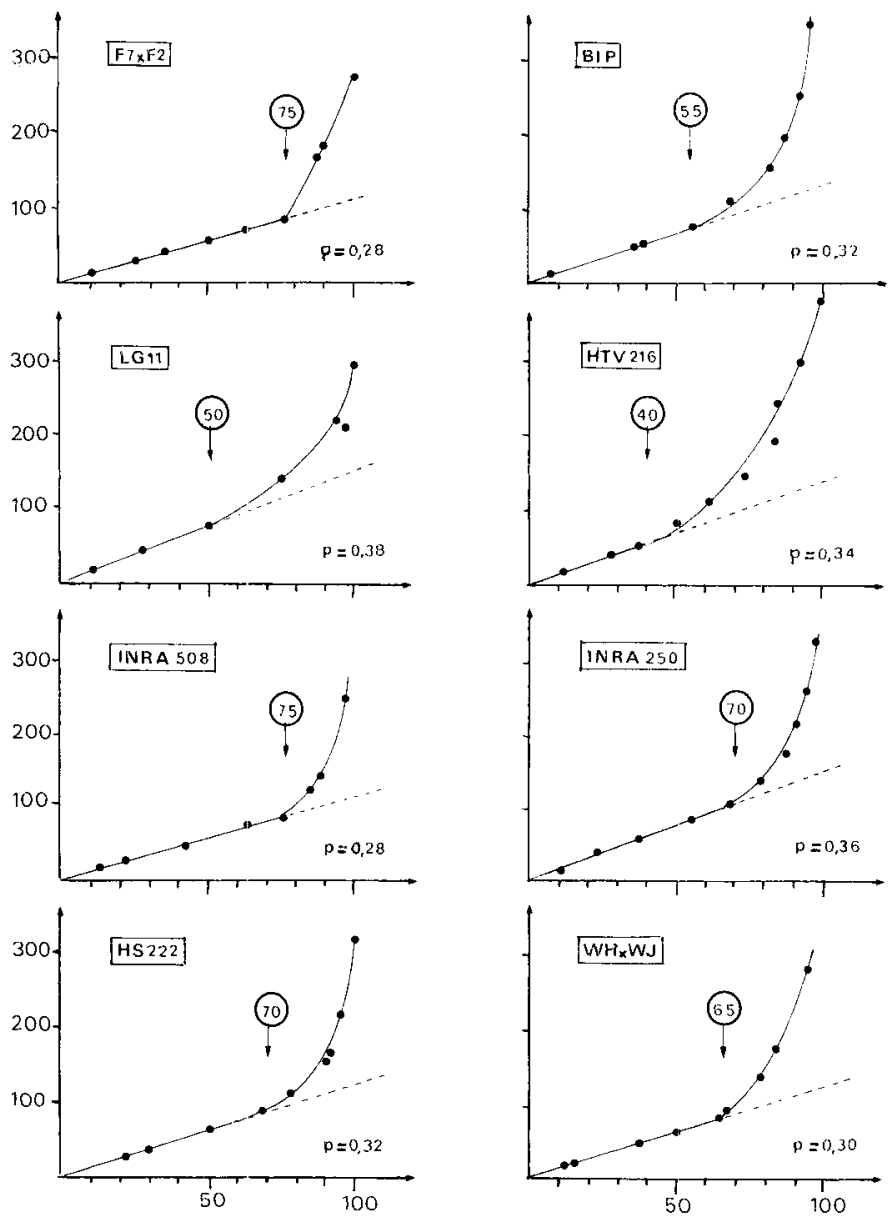

$\%$ des réserves disponibles utilisées

Figure 7

Corrélation entre le pourcentage des réserves utilisées et le poids atteint par la plantule. Comparaison entre 8 génotypes de maïs.

Dans le cercle: pourcentage des réserves utilisées à la fin de la période linéaire du graphique.

$p=$ pente de la droite pendant cette période.

Correlation between percent of reserves consumed and seedling weight. Comparison between eight maize varieties.

Circle: percent of reserves consumed at the end of linear phase of the graph.

$p$ : slope of the linear phase.

certaine quantité relative de réserves est nécessaire pour qu'une source métabolique se bâtisse qui doit prendre le relais de l'alimentation carbonée et énergétique de la jeune plante. Les 2 notions se rejoignent dans certains cas mais pas obligatoirement : ainsi, dans nos conditions expérimentales, les germinations qui présentent l'« efficacité d'utilisation » la plus élevée, puisque c'est très tôt que la croissance de la plantule acquiert son autonomie, sont celles fournies par HTV 216 (40 p. 100 des réserves).

Les pentes des droites initiales sont peu différentes les unes des autres. Cependant $\mathrm{F}_{7} \times \mathrm{F}_{2}$, INRA 508 et $\mathrm{W}_{\mathrm{H}} \times \mathrm{W}_{\mathrm{J}}$ ont des pentes significativement les plus faibles, INRA 250 la pente la plus forte. Ces différences révèlent des écarts dans la vitesse de consommation des réserves, dans l'efficacité des transferts, dans les capacités de croissance et d'utilisation des métabolites. Une approche plus complète de ces différents points nécessite une analyse plus fine des processus mis en cause. La différence des pentes est un fait brut qu'il est prématuré d'expliquer avec comme seul outil des données globales et pondérales qui sont rassemblées dans ces résultats.
Les données expérimentales, qui ne rendent compte que des variations de poids de matière sèche, ne peuvent, en aucun cas, apporter les éléments pour une compréhension fondamentale d'un processus physiologique. Les résultats exposés plus haut ne peuvent donc fournir des explications aux divers phénomènes, nombreux et très certainement en interactions qui se développent au cours des premiers jours de la vie active de la plante. Par contre, la référence aux variations du poids de la matière sèche présente un double avantage : elle fournit une information globale qui permet de mettre en évidence des phénomènes qu'une exploration plus fine, cellulaire et biochimique, peut ultérieurement et éventuellement expliciter, sinon expliquer; elle permet de développer des analyses assez fines de la croissance dans la mesure où les concepts développés et les formules appliquées sont soigneusement discutés comme ce fut fait par RADFORD (1967) et exploités par THORNLEY (1977).

Dans le cas présent, les données globales et pondérales obtenues ont été mises au service d'une comparaison entre semences de plusieurs génotypes. Il est capital de souligner que les différences observées portent sur le couple «génotype-semence » et il serait prématuré, voire erroné, de tirer des conclusions de classement intergénotypique rigoureux avant d'avoir pour chaque test une meilleure estimation de la variabilité due à l'origine des semences. Cependant la confrontation des résultats présentés permet une généralisation et une relativisation des phénomènes observés qui peut déboucher sur l'établissement de quelques lois générales ou de concepts opérationnels nouveaux.

\section{A. Généralisation des phénomènes observés au cours des premiers jours de la croissance des divers génotypes de mais}

Dans l'intervalle 0-12 j qui est celui de nos expériences, il est possible de définir, dans tous les cas, 4 phases caractéristiques :

- de 0 à $3 \mathrm{j}$, la germination proprement dite ;

- de 3 à $6 \mathrm{j}$, la diminution du taux initial de croissance de la plantulc jusqu'à acquisition d'un taux constant ;

- au $9^{\mathrm{e}}$ ou $10^{\mathrm{e}} \mathrm{j}$, la crise du «transfert de source»;

- au-delà de cette crise, l'établissement d'une croissance autonome.

Sur la germination proprement dite, c'est-à-dire du début de l'imbibition au pointement des organes de la plantule, le présent travail ne donne qu'une information globale.

Pendant les 5 ou 6 premiers jours, le taux de croissance initial de la plantule décroît alors que la disparition des réserves s'accélère. Ceci est un phénomène général que l'on retrouve chez tous les génotypes étudiés. L'antiparallélisme dans les variations de poids entre les 2 parties considérées montre que la relation entre la croissance pondérale de la plantule et l'utilisation des réserves n'est pas univoque : à un taux de croissance initial élevé correspond un taux de perte relativement faible, l'abaissement du premier correspondant à une accélération du second. Il semble donc que le coût du transfert de la source vers les zones d'utilisation s'ćlève, ce qui n'est pas pour surprendre quand on considère que les transports de substrats deviennent de plus en plus importants et que le travail accompli par le développement de la jeune plante exige une dépense énergétique accrue.

La continuité morphologique qui existe entre le scutellum et l'axe laisse supposer que, si une migration de substrats précoce s'établit, elle peut avoir à bon compte pour origine les tissus de réserve du scutellum. C'est bien ce qu'observe 
DURE (1960a) qui pense que pendant les 5 premiers jours c'est le scutellum seul qui, grâce à sa réserve lipidique, assure la croissance de l'axe. Cette perte serait compensée par une absorption des glucides solubles venant de l'albumen (EDELMAN et al., 1959). Pour INGLE et al. (1964) au contraire, c'est l'albumen qui fait essentiellement les frais du début de la croissance; ils estiment que la contribution du scutellum, qui contient cependant 80 p. 100 des réserves lipidiques de la semence, est négligeable.

Cette phase de la mise en place initiale de la plante au cours de laquelle l'apport photosynthétique ne semble pas jouer de rôle déterminant (fig. 7) met en jeu des processus multiples dont la résultante peut être la vigueur germinative ; cette notion a été discutée par WHALLEY et al. (1966). On constate que la structure-clé de cette phase est le scutellum qui joue un rôle complexe dont on peut résumer ici les différents aspects :

— Origine de la synthèse d' $\alpha$ amylase qui agira dans l'albumen, activité secrétrice (HORNING \& PETRIE, 1927 ; Dure, 1960b).

- Absorption des glucides solubles en provenance de l'albumen.

- Lieu de transfert de ces substrats vers l'axe (WHEELER \& HUMPHREYS, 1979 ; WHEELER et al., 1979 ; HUMPHREYS \& SMITH, 1980).

- Traitement des produits glucidiques en provenance de l'albumen (HUMPHREYS \& ECHEVERRIA, 1980).

- Production de glucides à partir de ses propres réserves lipidiques (BEEVERS, 1961).

- Exportation du saccharose (HuMPHREYS, 1978, 1981).

- Production de facteurs de croissance qui contrôlent le développement racinaire (TILTON, 1981).

L'amélioration des propriétés initiales et, par voie de conséquence, de la plante adulte devrait prendre en compte et approfondir nos connaissances sur la variabilité génotypique de cet organe de contrôle, le scutellum, placé à un poste clé au début de la croissance.

COOPER \& MACDONALD (1970) et plus récemment PinTo (1981) sur le blé ont bien montré que c'est aux environs du $10^{\mathrm{e}} \mathrm{j}$ que se situe la prise de relais entre les réserves qui s'épuisent et la photosynthèse qui s'établit. Parler du passage d'une hétérotrophie (avec source de carbone exogène à partir des réserves organiques) à une autotrophie (avec source de carbone minéral et photosynthétique) paraît, à la vue des résultats, une distinction un peu trop tranchée. L'autonomie de la jeune plante se fait graduellement et les interactions entre les 2 sources sont certainement puissantes avant que la succession soit complètement et définitivement établie.

Ce que nos résultats établissent c'est que ce passage provoque à un moment donné une crise de croissance, que cette crise est fugace et que son importance qualitative est variable suivant les génotypes et les semences. La répétitivité du phénomène d'un génotype à l'autre et le fait qu'il est marqué tant au niveau de la plantule qu'au niveau des réserves permettent d'établir qu'il s'agit d'une règle générale, une loi de croissance. Nous n'avons, pour l'instant, aucune information qui permette d'en expliquer les causes. Il est possible de faire l'hypothèse selon laquelle il y aurait un déphasage entre la vitesse de croissance des parties non photosynthétiques (= consommatrices) de la plantule et la croissance de l'appareil photosynthétique (= productrice); celui-ci n'aurait pas acquis sa pleine efficacité avant que le taux des réserves disponibles n'ait atteint un seuil minimum. S'il en était ainsi, une crise profonde ne serait pas forcément un signe défavorable pour l'avenir de la plante. En effet, cela signifierait qu'elle possède une forte aptitude à la croissance (transport, synthèse...) qui sera satisfaite par un système photosynthétique adulte ayant acquis sa pleine efficacité. Cependant, en tout état de cause, et dans l'intervalle de temps du présent travail, cette crise représente un retard, une sorte de manque à gagner qui, dans sa phase initiale de croissance, ne peut que défavoriser la mise en place de la plante. Si de plus, dans les conditions du champ, la crise correspond à une époque où l'environnement (thermique, hydrique ...) est défavorable, les conséquences néfastes peuvent être amplifiées.

\section{B. Quelques concepts opérationnels et quantifiables}

L'expérimentation simple sur des semences de 8 génotypes révèle des comportements qui, bien que différents dans l'amplitude des réactions, présentent un caractère répétitif. Cela permet de dégager des phénomènes constants qui traduisent des réactions physiologiques profondes irréductibles et générales; on peut donc parler ici de concepts. C'est parce que ces concepts peuvent être décrits expérimentalement, qu'ils ont une signification vitale pour la jeune plante et qu'ils peuvent servir à classer les semences que l'on peut dire qu'ils sont opérationnels. Enfin, les données expérimentales qui les concernent peuvent aboutir à des formulations simples exprimées en termes quantitatifs. Nous pensons que les résultats précédents permettent de dégager 5 de ces concepts qui, jusqu'à présent, ont peu attiré l'attention des observateurs.

\section{Les réserves utiles}

Nous avons vu que l'épuisement des substances accumulées dans la semence n'est pas complet. Il reste un résidu dont, pour l'instant, on ne sait rien quant à la nature chimique. On peut affirmer qu'il n'est pas seulement composé des parois et membranes résiduelles des parenchymes de réserve ; il renferme bien des molécules amylacées hydrolysables et exploitables. On peut penser que le rôle de ce résidu n'est pas négligeable dans l'établissement d'une microflore rhizosphérique. Il serait intéressant d'explorer les différences intergénotypiques quant à la composition de ce résidu.

\section{La régulation initiale du taux de croissance}

Il s'agit des phénomènes métaboliques qui contrôlent le taux de croissance au cours des 5 ou 6 premiers jours. Tous les graphiques montrent, pendant cette période, un abaissement du taux initial qui se traduit par une convexité des courbes (semi-log) dans cet intervalle de temps. On peut penser que les paramètres de cette courbe peuvent traduire des aptitudes métaboliques, différentes selon les génotypes, à contrôler les flux des molécules qui, partant des tissus de réserve, atteignent les sites d'utilisation.

\section{L'adaptation "autotrophique" ou mieux, le "relais énergétique »}

Nous avons vu qu'au moment ou s'installe un système photosynthétique efficace, vers le $10^{\mathrm{e}} \mathrm{j}$., et que les « réserves utiles »s'épuisent, la croissance de la jeune plante subit un choc qui est plus ou moins marqué selon les génotypes ; il est facile d'évaluer le retard pris. Il n'est pas certain que, dans tous les cas, ce retard ne soit pas compensé très rapidement. Cependant, dans une phase «sensible » de la vie de la plante, ce choc évident peut avoir des conséquences profondes si les conditions microclimatiques se révèlent limitantes, voire agressives. 


\section{Le bilan d'exploitation des réserves}

Ce bilan se traduit globalement par une perte de poids pendant une période plus ou moins longue (fig. 6). Nous avons vu qu'on peut estimer quantitativement la valeur cumulée de la perte au cours de cette période (tabl. 2). Le temps mis par la jeune plante pour rétablir un bilan positif peut être exprimé en un terme de précocité. Il serait intéressant de contrôler si la précocité de ce phénomène juvénile a quelque rapport avec la "précocité de maturité » telle qu'on a l'habitude de la définir chez la plante adulte.

\section{L'efficacité a'e l'emploi des réserves à des fins autotrophi- ques}

L'efficacité do l'utilisation des réserves pour la croissance pondérale de la jeune plante peut être donnée par le rapport entre la différence positive de poids de la plantule (gains) et la différence négative de poids des réserves (pertes) pendant le même intervalle de temps. Cette valeur qui n'a de signification simple qu'au début de la croissance, alors que celle-ci n'utilise que les réserves, peut exprimer le coût du transfert de molécules. Nous avons trouvé qu'il est variable selon l'origine génotypique des semences.

Mais il y a sans doute plus intéressant à considérer : c'est la quantité de réserves nécessaires à utiliser pour que, non seulement de la matière de plantule s'accumule (croissance pondérale), mais pour que des structures photosynthétiques efficaces s'installent (développement de l'équipement photosynthétique). C'est ce dernier aspect que nous proposons de considérer comme l'efficacité d'emploi des réserves à des fins autotrophiques. Le mode de représentation de la figure 7 permet de déceler des différences entre les génotypes ct de quantifier le phénomène.
En conclusion, il paraît possible, à la lecture des résultats et de l'exploitation qu'on peut en faire, de proposer 3 orientations de recherche:

- Il est évident que les observations globales sur la base de variations de poids de matières sèches imposent un approfondissement au niveau métabolique et cellulaire.

- Les donnécs présentes ont été obtenues pour des conditions microclimatiques contrôlées et affichées à un niveau arbitrairc. Si on vcut pousser la comparaison intergénotypique, il est indispensable d'étudier l'effet d'une ou plusicurs variables (température ...) et de s'assurer de l'indépendance vis-à-vis de l'origine des semences, c'est-àdire de leurs conditions d'obtention.

- L'ensemble de ce type de travail n'a de sens pratique et n'est efficace à des fins de sélection que dans la mesure où l'on pourrait établir des concordances entre les manifestations précoces et expérimentales et le comportement au champ à l'état juvénile (plantule). A terme, des corrélations avec la production finale des génotypes devraient être recherchées.

Reçu le 7 octobre 1982. Accepté le 11 avril 1983.

\section{REMERCIEMENTS}

Nous tenons à remercier Messieurs BONHOMME (Bioclimatologie, I.N.R.A.), M. Derieux (Amélioration des Plantes) et J.-L. PrIOUL (C.N.R.S.) pour les remarques judicieuses qu'ils ont bien voulu nous faire. Nous remercions Madame D. Gillion pour son aide technique.

\section{RÉFÉRENCES BIBLIOGRAPHIQUES}

Beevers H., 1961. Metabolic production of sucrose from fat. Nature, 191, 433-436.

Cooper C. S., MacDonald P. W., 1970. Energetics of early seedling growth in corn (Zea mays L.). Crop Sci., 10, 136-139.

Dure L. S., 1960a, Gross nutritional contribution of maize endosperm and scutellum to germination growth of maize axis. Plant Physiol., 35, 919-925.

Dure L. S., 1960b Site of origin and extent of activity of amylase in maize germination. Plant Physiol., 35, 925-934.

Edelman J., Shibko S. I., Keys A. J., 1959. The role of the scutellum of cereal seedlings in the synthesis and transport of sucrose. J. exp. Bot., 10, 178-189.

Horning R. H., Petrie A. H. K., 1927. The enzymatic function of mitochondria in the germination of cereals. Proc. R. Soc., B 102, 188-206.

Humphreys Th., 1978. A model for sucrose transport in the maize scutellum. Phytochemistry, 17, 679-684.

Humphreys Th., 1981. Sucrose-proton efflux from maize scutellum cells. Phytochemistry, 20/10, 2319-2323.

Humphreys Th., Echeverria E., 1980. Invertase and maltase in the free space of the maize scutcllum. Phytochemistry, 19, 189-193.

Humphreys Th., Smith R. C., 1980. Sucrose efflux from the maize scutellum. Ber. Deut. Bot. Ges., 93, 229-241.

Hunt R., 1980. Diurnal progressions in dry weight and short-term plant growth studies. Plant Cell Envircn, 3, 475-478.
Hunt R., Evans G. C., 1980. Classical data on the growth of maize : curve fitting with statistical analysis. New Phytol., 86, 155-180.

Ingle J., Beevers L., Hageman R. H., 1964. Mctabolic changes associated with germination of corn. I. Changes in weight and metabolites and their redistribution in the embryo axis, scutellum and endosperm. Plant Physiol., 39, 735-740.

Pinto Contreras M., 1981. Etude des coûts énergétiques de la croissance de plantules de blé (Triticum aestivum) lors du passage de l'hétérotrophie à l'autotrophie. Thèse d'Ingénieur-Docteur Sciences Agronomiques I.N.A.P.G., 1-12-1981.

Radford P. J., 1967. Growth analysis formulac. Their use and abuse. Crop Sci., 7, 171-175.

Tilton V. R., 1981. The influence of individual embryonic tissues on the morphology and development of Zea mays (Poaceae) germlings. Am. J. Bot., 68 (7), 980-993.

Thornley J. H. M., 1977. Growth, maintenance and respiration ; a reinterpretation. Ann. Bot., 41, 1191-1203.

Whalley D. B., McKell C. M., Green L. R., 1966. Seedling vigor and the early non-photosynthetic stage of seedling growth in grasses. Crop Sci., 6, 147-150.

Wheeler H., Humphreys Th., 1979. Properties of plasmalemma ATPase of the maizc scutcllum. Phytochemistry, 18, 555-560.

Wheeler H., Humphreys Th., Aldrich H., 1979. Localization of a phosphatase (ATPase) on the plasmalemma of the maize scutellum. Phylochemistry, 18, 549-554. 